\title{
A community virtual ward model to support older persons with complex health care and social care needs
}

This article was published in the following Dove Press journal:

Clinical Interventions in Aging

26 June 2017

Number of times this article has been viewed

\section{Lewis' \\ Z Moore' \\ F Doyle ${ }^{2}$ \\ A Martin ${ }^{3}$ \\ D Patton' \\ LE Nugent'}

'School of Nursing and Midwifery, Royal College of Surgeons Ireland, ${ }^{2}$ Department of Psychology, Royal College of Surgeons in Ireland,

${ }^{3}$ Beaumont Hospital, Dublin, Ireland
Correspondence: C Lewis School of Nursing and Midwifery, Royal College of Surgeons in Ireland, $123 \mathrm{St}$ Stephen's Green, Dublin 2, Ireland Tel +353 I 860263702 Email clarelewis@rcsi.ie
Background: Globally the older population is increasing rapidly. As a result there is an increase in frail older persons living within the community, with increased risks of a hospital admission and higher mortality and morbidity rates. Due to complexity of care, health care professionals face challenges in providing effective case management and avoiding unplanned admissions to hospital. A community virtual ward (CVW) model was developed to assist health care professionals to support older persons at home during periods of illness and/or functional decline.

Methods: A quantitative observational study was conducted to examine if a CVW model of care reduced unplanned hospital admissions and emergency department (ED) presentations in 54 patients over a 12-month period. The sign-rank test examined matched data on bed days, ED presentations, and unplanned hospital admissions pre- and post-CVW implementation. Other risk factors for admission to hospital were examined using the Mann-Whitney test preand post-CVW admission, including falls, living alone, and cognition. Correlations between hospital admission avoidances and unplanned hospital admissions and ED presentations were tested using Spearman's $\rho$ test.

Results: There was a reduction in ED presentations post-CVW admission $(P<0.001)$, and median unscheduled admissions were reduced $(P=0.001)$. Those living alone had a lower number of ED presentations (median 0.5, interquartile range $0-1$ ) prior to admission in comparison to those living with a caregiver, with no differences observed during admission to CVW. For those who experienced a fall during CVW admission, the odds ratio (OR) of requiring long-term care doubled for each extra fall ( $\mathrm{OR}=2.24,95 \% \mathrm{CI} 1.11$ to $4.52, P=0.025)$. Reduced cognition was associated with an increased risk of ED presentations $(\rho=0.292, P<0.05)$ but not associated with increased risks of unplanned hospital admissions $(\rho=0.09, P=0.546)$. There were no significant correlations seen between admission avoidance and the number of unplanned hospital admissions or ED presentations.

Conclusion: Through an integrated approach to care, a CVW model in the care of older persons can reduce ED presentations and unplanned hospital admissions.

Keywords: community virtual ward, case management, integrated care, older persons' care, complex care

\section{Introduction}

In the last decade, there has been a global increase in life expectancy with those in the 60-year age group expected to live an additional 20 years. ${ }^{1}$ In North America, by 2050 , it is predicted that $4.4 \%$ of the total population will be $\geq 85$ years, with men living to an average age of 82.2 years and women 86.2 years. $^{2}$ In Europe, $18 \%$ of the population is $>65$ years increasing by $2.3 \%$ in comparison to 10 years ago. ${ }^{3}$ 
Similarly, in America, life expectancy has increased for both genders, by $\sim 10$ years over the last decade. ${ }^{3}$ Likewise, in the Republic of Ireland, the aging population is growing within the octogenarian age group, with a $4 \%$ increase per annum. ${ }^{4}$ As a result, there are increases in emergency department (ED) presentations and hospital overcrowding, with hospitals working at $100 \%$ capacity. ${ }^{4}$ As part of government recommendations community services are developing, and this includes nursing posts such as a clinical case manager (CCM) for older persons, to provide clinical case management to older persons with complex health care needs within the community. ${ }^{4,5}$ The role focuses on clinical care and coordination of services, with a patient-centered focus to support people within the community, reducing reliance on secondary care services. ${ }^{5,6}$ However, providing effective case management can present challenges as health care professionals (HCPs) work within their own silos of care and adopt a reactive approach to care. ${ }^{6-8}$ Furthermore, risk stratification to identify those at risk of a hospital admission, to inform decision making, has been viewed as inadequate in those with complex care needs. ${ }^{9}$

\section{Community virtual wards}

To assist the HCPs with risk stratification and decision making in those with complex care needs, community virtual ward (CVW) models have been developed internationally. ${ }^{10-13}$ However, there is a dearth of published research in this area limited to studies from the UK, Hong Kong, and Canada. ${ }^{10-14}$ A CVW model provides a framework of care to assist the HCPs in determining main risks and informs decision making with regard to prioritization of care, timely mobilization of services, and monitoring to support patients to remain at home. ${ }^{11,12,15}$ This approach to care allows for targeted specific interventions that are proactive, rather than reactive, within a framework of case management. ${ }^{15}$ The CVW models in the UK, Canada, and Hong Kong have operated differently depending on service infrastructure and focus of care. ${ }^{10-15}$ In the UK, the model was embedded within primary care with a dedicated CVW team led by a general practitioner (GP) and overseen by a community matron, a nursing post that provides case management for individuals with long-term conditions with a focus on self-management. ${ }^{10,15}$ Patients were selected following risk stratification using an electronic predictive risk tool (the PARR or a combined model) within the GP practices, basing risk on the number of hospital admissions within the last $6-12$ months and comorbidities. ${ }^{10}$ Therefore, the majority of referrals to the CVW were from community-dwelling settings admitted to the CVWs for on average of 7 months. In Canada, the model focused on patients post-discharge from hospital, and risk was stratified using an electronic tool (LACE) that compared length of stay, type of hospital admission, comorbidities, and ED visits in the last 6 months. ${ }^{11}$ Similar to the UK, it was led by a primary care physician with a case manager and nurse practitioner working out of a community care access center, and patients post-discharge from hospital were admitted to the CVWs for several weeks.

In Hong Kong, the model of care centered around patients post-discharge from hospital with two dedicated teams within the hospital and community. ${ }^{12}$ This included link nurses within the hospital setting working with consultant geriatricians focusing on discharge preparation, including medication management and symptom recognition, and referring on to the CVW team for a period of 3 months. The community team provided home-based therapy and follow-up facilitating day hospital review if required. The CVWs were overseen by a case manager that rotated between a nurse practitioner, occupational therapist, physiotherapist, and social worker. ${ }^{12,16}$ Patients were risk stratified using an electronic predictive risk tool (HARRPE), determining a hospital admission 28 days after the acute event. The HARRPE tool considers 14 variables of risk including socio-demographics, prior hospital admissions, comorbidities, and acuity of the recent hospital event. ${ }^{12}$

Results from the CVWs discussed have been variable and this may be due to model delivery. In the UK, one study examined retrospective data on over 900 patients using sample matching techniques to compare a control group with those admitted to the CVWs. ${ }^{10}$ The results indicated no significant reductions in ED presentations and unplanned hospital admissions. However, models were based on primary care with no evidence of formal integration with secondary care, or pathways for acute care access bypassing the ED. ${ }^{10}$ Similarly, a randomized controlled trial in Canada, including over 1,000 patients, showed no significant reductions in ED visits at 30 days, 90 days, 6 months, and 1 year. ${ }^{11}$ Researchers reported difficulties communicating with primary care physicians and the use of varying electronic systems as barriers to care provision. Further barriers included placement of the team varying from the hospitals where patients were discharged, resulting in difficulties accessing information or engaging with clinicians previously involved in patient care. ${ }^{11}$ To date, Hong Kong has been the most successful model. Through a randomized control trial of 39 patients in both intervention and control groups, they observed a significant reduction in the number of unplanned admissions at 90 days. ${ }^{12}$ However, they observed no significant difference in ED presentations 
between the groups. Researchers reported that success of this model may be reflected in the protocol-driven integrated approach to care working across primary and secondary care with intensive home care delivered by a multidisciplinary team (MDT). ${ }^{12,16}$ Furthermore, commencement of interventions prior to hospital discharge is seen to improve outcomes within the community. ${ }^{11}$

All of the models discussed have focused on long-term conditions, most notably, chronic obstructive airways disease and chronic heart failure, as well as renal disease. ${ }^{10-15}$ The emphasis has been on self-management strategies of care, as well as re-enablement over an average period of 8-12 weeks. ${ }^{11,12}$ In Hong Kong, the CVW team visited patients up to four times per week. ${ }^{12}$ This may be unrealistic given the increase in the older population and the numbers of frail older persons living within the community. Furthermore, within the studies, there is limited evidence of how a CVW assists in the general care of older persons living in the community. This includes dementia care as the prevalence of dementia is increasing globally and models supporting case management and integrated care are required ${ }^{17}$ Hence, the CVW in this study considers the general frail older population rather than specific chronic illnesses.

\section{Community virtual ward, North Dublin}

Prior to the CVW, patients in North Dublin were not formally risk stratified and often had multiple service providers involved. Therefore, a number of potential issues were identified. These issues included prioritizing care within a high-risk group, capturing events to inform decision making, identifying appropriate service interventions and responses to these, and engaging with key HCPs within primary and secondary care to support older persons to remain at home. A cohesive approach to case management to inform decision making was needed to ensure proactive care planning and also to determine when a patient was stable for discharge to usual care, provided by the primary care team. Therefore, the CVW model in North Dublin was developed expanding beyond chronic disease management, with a focus on the care of older persons with complex health care and social care needs. The model provided a framework for CCM to prioritize those who were at risk of a hospital admission, or a further event, due to either functional decline or clinical presentation.

\section{The CVW model}

The model was set up to work within existing resources within primary and secondary care through an integrated approach to older persons' care overseen by a CCM who is a senior nurse working across primary and secondary care. This was key in providing timely service interventions and follow-up, as well as access to the day hospital if there was evidence of clinical or functional deterioration and/or organization of a planned admission to hospital.

\section{Functional decline and increasing social supports}

The majority of referrals to the CVW were due to functional or clinical decline precipitated by either cognitive or physical deterioration impacting on activities of daily living as well as increasing need in social supports. ${ }^{18-20}$ From a national and international viewpoint, functional decline with risks of hospital admission is grouped under the umbrella term of frailty. ${ }^{5,21-23}$ This can be divided into physical frailty, cognitive deterioration, dementia, depression, functional decline, polypharmacy, nutrition, comorbidities, and social isolation. ${ }^{5,21,22}$

\section{Frailty}

Frailty was assessed using the Rockwood Clinical Frailty Index $\mathrm{Scale}^{24}$ at initial presentation and on transfer between CVWs.

\section{Level of dependence}

In determining the level of dependence, the Barthel Clinical Index ${ }^{25}$ was used at the time of admission and on transfer.

\section{Risk of hospital admission}

To estimate risk of a hospital admission, the identification of seniors at risk (ISAR) tool was used, with a score of $\geq 2$ seen as higher risk of a hospital admission. ${ }^{26}$

\section{Cognition}

The mini mental state examination (MMSE) was used to assess cognition on admission and transfer between CVWs. This tool assists in determining overall cognition at baseline and as a continual measure of cognition over time in both hospital and community settings. ${ }^{27,28}$ The tool assesses for orientation, attention, recall, and language, with a cutoff score of 24 out of an overall score of 30 points. $^{28}$

\section{Levels of risk}

The CVW operated on a traffic light system of red (high risk), amber (moderate risk), and green (low risk), with types of service interventions and monitoring depending on the selection of ward admission. There were 10 beds in the red (high risk) virtual ward (VW), 10-15 in the amber 
(moderate risk) VW, and 15 in the green (low risk) VW, reflecting upon the maximum case load of the CCM. Patients moved through the VWs eventually transferring to the green VW and were discharged to the care of the primary care team once considered stable with no events for a 4-6 week period and supported with current home care supports. Once discharged, a community public health nurse, a member of the primary care team, would review the patient 4 weeks post discharge from the CVW to re-asses.

\section{The CVW admission, beds, and referrals}

Patients were admitted to the CVW for $\sim 3-7$ months depending on the response to service interventions. Referrals were received from a consultant geriatrician, following the MDT assessment, from the day hospital, outpatient gerontology clinics, or prior to hospital discharge.

\section{Clinical case management}

The CCM reviewed the patients within their own home, completing an assessment to determine level of frailty and acuity of event coupled with cognition and current social supports. Determining the risk of an admission to hospital was based on the type and number of events, and if they had occurred in the last 30 days. ${ }^{10-15}$ The event and overall assessment were compared as part of the triage process to determine if a VW admission was required and the selection of the VW. The day hospital within the locality was the central hub of the model, with a hub-and-spoke approach to care connecting services involved in the care of the patient and family, which includes service interventions and monitoring. Patients were monitored with a combination of home visits and telephone consultations. A combined approach to care using home visits and follow-up telephone consultations has proven to be effective in supporting patients and caregivers at home in improving the quality of life and self-care management and reducing ED presentations. ${ }^{18,19,29}$ In contrast, there is evidence that home visits alone are not effective in reducing hospital readmissions. ${ }^{30}$

\section{Interventions}

Primary interventions were determined by the presenting events based on the assessment and there were five main pathways of care. These included clinical, functional involving rehabilitation and/or re-enablement, social support, behavioral and palliative. The range of interventions managed within the community were facilitated by the primary care teams including the CCM, GP, public health nurse, physiotherapist, occupational therapist, social worker, and pharmacist. The CCM also worked closely with the consultant geriatrician to determine if patients required additional input from a specialist therapist-led integrated care team and day hospital input if care needs exceeded that of the primary care team services. The CCM worked with the GP and geriatrician to determine if patients required additional input from secondary care specialists in cardiology, respiratory, and diabetes as well as community specialists in psychiatry and palliative care. To support the CCM role and as part of the model of care, it was agreed that the CVW would have access to a nurse-led community intervention team to support home-based interventions such as intravenous (IV) therapies and clinical monitoring during periods of deterioration. It was also agreed locally that, if an increased social support was required within the home, this would be prioritized.

\section{Materials and methods Study aims}

The primary aim of the study was to determine if unplanned hospital admission and ED presentations could be reduced through a CVW model.

The secondary aims examined the following:

1. If living alone increased the risks of a hospital admission or ED presentation.

2. If cognitive impairment significantly increased the risk of hospital admission during the CVW admission, and the relationship between admission avoidances and additional hospital inpatient admissions.

3. The relationship between falls during the CVW admission and prediction for long-term care (LTC) was also examined.

\section{Research questions}

The primary research question was regarding the evidence of a reduction in unplanned hospital admissions and ED presentations for patients admitted to the CVW.

\section{Study design}

A quantitative observational study examined the number of hospital admissions and ED presentations 6 months prior to admission to the CVW and on discharge as well as the number of bed days pre- and post-CVW admission. ${ }^{31}$ The risk of hospital admission was measured on admission to the CVW. Hospital admission activity was examined in those living alone or with a caregiver 6 months prior to admission and on discharge from the CVW. The number of falls 3 months prior to admission and during the CVW admission was compared in relation to outcomes. Baseline cognition and number of unplanned admissions and ED presentations 
were measured prior to CVW admission and on discharge. Baseline demographics including age, gender, social history, comorbidities, level of frailty, function, cognition, and falls were collected at baseline. The number of falls was measured following each transfer between the CVWs and on discharge. This was a single-centered study using a convenience sample referred by consultant geriatricians in the locality. Therefore, the model of care was developed as part of clinical practice to support the CCM role rather than a controlled research study, hence there was no control group.

\section{Sample}

A convenience sample of older persons $\geq 65$ years was selected as referrals were directly received from gerontology services.

\section{Ethical approval}

Ethical approval was obtained from the research ethics committee at the Royal College of Surgeons in Ireland, on April 21, 2016. The ethical approval enabled the researchers to include retrospective data on patients admitted to the CVW. Participants in the study were known to the CCM and the geriatrician. To protect patient confidentiality and anonymity, each case was coded on entry to the CVW. Informed consent was obtained retrospectively from patients who were admitted to the CVW or, if unable, assent was obtained by a family member or legal representative.

\section{Data collection}

Data were collected from November 2014 to November 2015, $(\mathrm{N}=54)$. Descriptive statistics pertaining to baseline demographics, comorbidities, social supports, level of frailty using the Rockwood Clinical Frailty Index Scale, ${ }^{22,24}$ function using the Barthel Index, ${ }^{25}$ cognition using the MMSE, ${ }^{27}$ and falls were captured prior to admission and during admission to the CVW. The ISAR tool measured risk of a hospital admission at the time of admission to the CVW. ${ }^{26}$ The number of bed days, ED presentations, and unplanned hospital admissions were collected pre- and post-CVW admission. The number of admission avoidances was recorded during the CVW admission, as well as the number of falls and older persons transferring from the community into LTC.

\section{Data analysis}

All statistical analyses were performed using the STATA software package version 14 (StataCorp LP, College Station, TX, USA). The sign-rank test measured bed days pre- and post-CVW admission, as well as tested if there was a positive reduction in unplanned hospital admissions and ED presentations. ${ }^{32}$ The Mann-Whitney test examined hospital activity in those who lived alone or with someone, comparing this on admission to and discharge from the CVW. ${ }^{32}$ Spearman's $\rho$ test measured the correlations between admission avoidances and subsequent unplanned hospital admissions and ED presentations, as well as the risks of falls pre- and post-CVW admission and admission to LTC. ${ }^{32}$ The test also looked for correlations between a decrease in cognition and the number of unplanned hospital admissions and ED presentations pre- and post-CVW admission.

\section{Results}

A total of 54 patients were included in the study, who were known to the CCM, geriatrician, GP, public health nurse, allied health care professionals, pharmacist, and home care agencies.

\section{Demographics}

The average age of patients admitted to the CVW was 81.6 years with more women (68\%) than men (31\%). Most of them were moderate to severe frail with a high risk of a hospital admission, and thus all were initially admitted to the red CVW. Risk scores are presented in Table 1. A total of $77 \%$ of patients lived alone with $37 \%$ of patients receiving informal support from family caregivers who were instrumental in monitoring and supporting service interventions in addition to 629.5 home care hours. On admission, patients were taking on average $\geq 11$ medications and living with two or more comorbidities. The most common comorbidities were dementia, cardiovascular disease, hypertension, cerebral vascular disease, diabetes, and chronic obstructive airways disease. The demographic characteristics of patients included in this study are presented in Table 2.

\section{Length of stay in CVW}

Response to service interventions over time guided transfer to lower levels of the CVW, either amber or green. The average length of stay for patients was between 3 and 7 months due to level of frailty and clinical needs. This reflects the international evidence around length of stay within a CVW framework. ${ }^{9,15}$

Table I Risk scores on admission to community virtual ward

\begin{tabular}{ll}
\hline Risk scores & Results (mean \pm SD) \\
\hline Frailty & $6.7 \pm 0.86$ (moderate-severe) \\
Functional dependence & $11.2 \pm 4.7$ (moderate-severe) \\
Hospital admission risk & $4.85 \pm 0.88$ (high risk) \\
Cognition & $19.2 \pm 5.10$ (moderate) \\
\hline
\end{tabular}


Table 2 Demographic characteristics

\begin{tabular}{ll}
\hline Characteristics & Results \\
\hline Age, mean \pm SD & $81.6 \pm 5.7$ years \\
Male, $\mathrm{n}(\%)$ & $3 \mathrm{I}$ \\
Female, $\mathrm{n}(\%)$ & 68 \\
Main comorbidities, $\mathrm{n}(\%)$ & \\
Dementia & 42 \\
Cardiovascular disease & $1 \mathrm{I}$ \\
Hypertension & 27 \\
Cerebral vascular disease & 14 \\
COPD & 16 \\
Social supports & \\
Living with a carer, $\mathrm{n}$ & 77 \\
Living alone, $\mathrm{n}$ & 37 \\
Informal care, $\mathrm{n}$ & 86 \\
Home care package hours, mean \pm SD & $12.6 \pm 7.51$ \\
\hline
\end{tabular}

\section{Hospital admissions and ED presentations}

Hospital bed days assessed using the sign-rank test showed a decrease in the number of bed days after interventions through the CVW (bed days pre-CVW: median 23, interquartile range [IQR] $0-68$; bed days post-CVW: median 0 , IQR $0-14 ; z=3.67, P<0.001)$. The number of ED presentations was lower post-CVW admission (ED presentations pre-CVW: median 1, IQR 0-2; ED presentations post-CVW: median 0, IQR $0-1 ; z=4.52, P<0.001)$. The number of unscheduled admissions was also reduced in comparison to the number of unplanned hospital admissions prior to the CVW interventions (unplanned hospital admissions preCVW: median 1, IQR 0-1; unplanned hospital admissions post-CVW: median 0, IQR 0-0; $z=4.79, P=0.001$ ).

\section{Admission avoidance}

Admission avoidance was determined by the clinical intervention provided normally resulting in a hospital admission such as management of delirium, IV antibiotics, and sub-cutaneous (sub-cut) fluids. Also the patients' previous hospital admission presentation was examined and compared to presenting acute events managed through the CVW. The greatest number of interventions and admission avoidances occurred in the red (high risk) CVW within the first 4 weeks of admission. Clinical events managed in the community as part of admission avoidance covered a spectrum of care including the management of delirium, behavioral and psychological symptoms of dementia, and functional decline providing a home-based exercise and functional program or admission to a community rehabilitation bed. Other significant issues included pain management affecting function, symptomatic polypharmacy, dehydration requiring the use of sub-cut fluids, and decompensating heart failure requiring IV diuretics as an outpatient. Other episodes of care managed through the VWs included exacerbations of COPD, chest infections, and cellulitis requiring IV antibiotic therapy in the home or managed within a step-up community bed. The relationship between admission avoidances and ED visits was examined using Spearman's $\rho$ test. However, there was no significant correlation between these variables at either time point (pre-CVW: $\rho=0.14, P=0.315$; post-CVW: $\rho=0.163, P=0.239)$.

\section{Living alone or with a caregiver}

Prior to admission to the CVW, there was evidence that those living alone had fewer hospital admissions (bed days: median 0, IQR 0-19) than those living with someone (bed days: median 47, IQR 14-83; $z=2.5, P=0.012$ ). In addition, those living alone had a lower number of ED presentations (ED presentations: median 0.5, IQR 0-1) than those living with someone (ED presentations: median 1, IQR 1-2; $z=1.99, P=0.047)$. There were no differences observed in the number of unplanned hospital admissions or ED presentations in those living with someone or living alone post-CVW admission.

\section{Falls and cognition}

There was evidence that falls prior to admission to the CVW were correlated with falls post-CVW admission ( $\rho=0.303$, $P=0.026)$. For those who experienced $\geq 1$ fall during the CVW admission, the odds ratio (OR) of requiring LTC doubled for each extra fall the person experienced $(\mathrm{OR}=2.24$, 95\% CI 1.11 to $4.52, P=0.025$ ).

Reduced cognition scores were not associated with an increase in unplanned hospital admissions ( $\rho=0.09, P=0.546$ ), but there was a positive correlation between an increase in ED presentations and reduced cognitive scores $(\rho=0.292$, $P<0.05)$.

\section{Discussion}

This study demonstrates that a CVW model for the care of older persons with complex health care and social care needs can assist in reducing the number of ED presentations and unplanned hospital admissions. Similar to the CVW model in Hong Kong, ${ }^{12}$ an integrated approach to care, working with specialists in primary and secondary care was key in supporting older persons at home during critical periods of illness and functional decline. Increased nursing input was key to this, in addition to health care assistant support in the care of older persons during periods of clinical and functional 
decline. Access to a nurse-led community intervention team was paramount in providing therapies such as sub-cut fluids, sub-cut symptom-control medications, and IV antibiotics within the home, as well as the clinical reassessment out of hours during subacute events. Prioritization of home care supports with access to home care package ensured that caregivers were supported and also provided supervision and support with medication management, hydration, nutrition, and personal care. Access to a day hospital was paramount for complex care when care needs exceeded that of primary care services and this acted as a central hub within this study, providing ambulatory care and also an outreach service for those unable to attend due to illness and/or functional decline. The function of the day hospital is to provide coordination, support, and integration of care. ${ }^{5}$ Therefore, the CVW provided an extension of the specialist care within the home and monitoring of a high-risk group. Furthermore, access to an integrated specialist therapist-led team working across hospital and community provided home-based programs of care for those on the cusp of a hospital admission due to functional decline. Similar to previous work, ${ }^{12}$ this study demonstrated that providing a CVW model of care to those at high risk of a hospital admission through an interdisciplinary approach can reduce hospitalization and ED presentations.

However, unlike previous studies, ${ }^{10-15}$ rather than focusing on chronic disease and self-management strategies, this study focused on the care of older persons and management of clinical and/or functional decline. Unlike the CVW model in Hong Kong, ${ }^{12}$ this study was not protocol-driven in terms of follow-up, but rather individualized based on presenting events and responses to service interventions. However, a combination of home visits and telephone consultations proved successful, rather than home visits alone. This supports the findings of previous research reporting that community nursing home visits alone had no impact on hospital readmissions. ${ }^{30}$

Prior to the CVW admission, those living alone spent less time in hospital than those living with a caregiver and also had less ED presentations. Caregiver burnout and stress has been associated with a greater risk of hospital admissions and ED presentations. ${ }^{33}$ Therefore, caregiver support is seen as impacting on a reduction in hospital admissions supporting frail older persons to remain at home. ${ }^{33}$ Interestingly, in this study, there were no differences in the number of ED presentations and unplanned hospital admissions for those living alone or with a caregiver during the CVW admission. This is possibly due to specialist MDT involvement across primary and secondary care, and social supports with caregiver supports during CVW admission. This finding is supported by previous work through the Program of All-Inclusive Care for the Elderly (PACE) model..$^{33}$ PACE is an integrated care model working across acute and primary care focusing on care coordination, early intervention, and support for patients and caregivers within the community, reporting a reduction in hospital bed days used. ${ }^{20}$ Therefore, this study suggests that a CVW model of care using an integrated approach results in better outcomes for frail older persons whether living alone or with a caregiver.

There is increasing evidence that interventions that result in admission avoidances can reduce the number of ED presentations and unplanned hospital admissions in a frail older population. ${ }^{33,34}$ However, in this study, no significant correlation was found between admission avoidance and the number of ED presentations and unplanned hospital admissions. Nevertheless, there were reductions in ED presentations, unplanned hospital admissions, and bed days overall. Consequently, although there was no significant association with admission avoidance in our small sample, it is likely that hospital admissions could have been higher.

As the prevalence of dementia is increasing, it is paramount that models of care within the community evolve to support individuals within their own homes. ${ }^{17}$ In this study, reduced cognition was not significantly associated with an increase in the number of hospital admissions. However, there was a correlation between reduced cognition and higher number of ED presentations. Closer monitoring of patients with advancing dementia, with identification of red-flag clinical presentations and early interventions, as well as a specialist follow-up, may provide explanations for reduced hospitalization; however, ED presentations remain a high risk for this group. Hence, evolving the CVW model of care for patients living with dementia during critical periods of illness and/or functional decline to avoid ED presentation may reduce this and avoid unnecessary hospital admissions and delay the need for LTC.

Falls present major challenges within health care in terms of hospital admissions and a risk of mortality or further morbidity to the individual. ${ }^{35}$ In this study, those who experienced a fall prior to admission to the CVW had an increased risk of a further fall during admission. However, it is important to note that the majority of patients were severely frail, and evidence suggests that this in itself increases the risk of falls..$^{23,35,36}$ Additional findings in our study indicated that the need for LTC almost doubled for every additional fall experienced during admission. Further functional decline can be seen up to 1 year following a fall, therefore potentially 
increasing the risk of institutionalization. ${ }^{36}$ The findings of our study provide insight into future care planning and proactive case management increasing preventative falls interventions to avoid or delay the need for LTC. Furthermore, it provides a trigger with regard to early discussions regarding LTC with the patients and caregivers if they do not respond to interventions provided.

\section{Limitations}

This study has a number of limitations, noteworthy of them are a small sample size, the lack of a comparison group, and the use of retrospective data. Also, healthrelated quality of life was not examined through this model of care. This study did not have a comparison group as the model of care was developed within clinical practice to support the role of the CCM reducing pressures on secondary care services. Nonetheless, the findings from this study are consistent with previous research ${ }^{12}$ and add further evidence to the benefits of a CVW model from a different perspective. Further research expanding on this study's findings is required to determine the overall effectiveness of CVWs in a larger population including comparison groups and health-related quality of life.

\section{Conclusion}

There have been a number of versions of CVWs that are either embedded in primary care with GP practices as the central hub, an outreach approach to care, or integration with primary and secondary care services. ${ }^{10-15}$ These models, to date, have focused on chronic disease management rather than older persons' care, including dementia care, and predominantly targeted patients post-discharge from hospital. As care needs of the aging population change and increase, the CVW models must evolve moving beyond chronic disease and post-discharge care to holistic management of older persons' care within primary care prior to a hospital admission.

This study worked within existing resources and streamlined care practices and pathways of care working closely with acute services including specialist gerontology and primary care. However, as the aging population is increasing exponentially, with greater clinical and functional needs, this may not be a sustainable approach with the potential of becoming a reactive care model. Therefore, similar to previous models, ${ }^{10-15}$ dedicated integrated specialist CVW teams working across primary and secondary care in the care of older persons may offer a solution to overstretched EDs and unnecessary hospital admissions.

\section{Acknowledgments}

The researchers thank the School of Nursing and Midwifery, Royal College of Surgeons in Ireland, the Nursing and Midwifery Planning and Development Unit, North Dublin, the Beaumont Hospital Specialist Gerontology Services including the Day Hospital, the Health Service Executive Community Health Care Organisation (Area 9), Mary McDermott, Director of Nursing for Public Health, Jean Whelan, Assistant Director of Nursing Public Health, and Gwen Regan, Practice Development Coordinator, North Dublin, for their support for this initiative. This paper was presented at the Health Service Executive National Clinical and Integrated Care Forum 2016 held at Dublin, Ireland, on October 18 at a breakout session with interim findings.

\section{Disclosure}

The authors report no conflicts of interest in this work.

\section{References}

1. United Nations Committee. World Population Ageing. United Nations Committee; 2013. Available from: wwwunorg/en/development/desa/ population/publication/pdf/ageing/WorldPopulationAgeing2013. Accessed March 10, 2017.

2. Ortman JM, Velkoff VA, Hogan H. An Aging Nation: The Older Population in the United States: Population Estimates and Projections. Maryland, US: US Department of Commerce; 2014:25-114.

3. Eurostat. European Statistics. Eurostat; 2015. Available from: eceuropaeu/eurostat/data/database. Accessed March 10, 2017.

4. Department of Health. Emergency Department Task Force Report. Department of Health; 2015. Available from: www.healthgovie/ publications/emergency-departmenttask-force-rerport. Accessed August 28, 2015.

5. Health Service Executive. National Clinical Care Programme for Older People: Specialist Geriatric Services Model of Care. Dublin: Health Service Executive \& Royal College of Surgeons Ireland; 2012: $1-95$.

6. Hudson AJ, Moore LJ. A new way of caring for older people in the community. Nurs Stand. 2006;20(46):41-47.

7. Gravelle H, Dusheiko M, Sheaff R, et al. Impact of case management (Evercare) on frail elderly patients: controlled before and after analysis of quantitative outcome data. BMJ. 2007;334(7583):31.

8. Bentley A. Case management and long-term conditions: the evolution of community matrons. Br J Community Nurs. 2014;19(7):340, 342-345.

9. Lewis G, Wright L, Vaithianathan R. Multidisciplinary case management for patients at high risk of hospitalization: comparison of virtual ward models in the United Kingdom, United States, and Canada. Popul Health Manag. 2012;15(5):315-321.

10. Lewis G, Georghiou T, Steventon A, et al. Impact of virtual wards on hospital use: a research study using propensity matched controls and a cost analysis. National Institute for Health Research Service Delivery and Organisation Programme. 2013;(2013):1-157.

11. Dhalla IA, O'Brien T, Moora D, et al. Effect of a postdischarge virtual ward on readmission or death for high-risk patients: a randomized clinical trial. JAMA. 2014;312(13):1305-1312.

12. Leung DY, Lee DT, Lee IF, et al. The effect of a virtual ward program on emergency services utilization and quality of life in frail elderly patients after discharge: a pilot study. Clin Interv Aging. 2015;10:413-420. 
13. Raphael MJ, Nadeau-Fredette AC, Tennankore KK, Chan CT. A virtual ward for home hemodialysis patients - a pilot trial. Can J Kidney Health Dis. 2015;2:37.

14. Jones J, Carroll A. Hospital admission avoidance through the introduction of a virtual ward. Br J Community Nurs. 2014;19(7):330-334.

15. Lewis G, Vaithianathan R, Wright L, et al. Integrating care for highrisk patients in England using the virtual ward model: lessons in the process of care integration from three case sites. Int J Integr Care. 2013; 13:e046.

16. Lin FO, Luk JK, Chan TC, Mok WW, Chan FH. Effectiveness of a discharge planning and community support programme in preventing readmission of high-risk older patients. Hong Kong Med J. 2015;21(3): 208-216.

17. Cahill S, O'Shea E, Pierce M. Creating Excellence in Dementia Care A Research Review for Ireland's National Dementia Strategy. Ireland: Irish Centre for Social Gerontology; 2012:1-152.

18. Coleman EA, Parry C, Chalmers S, Min SJ. The care transitions intervention: results of a randomized controlled trial. Arch Intern Med. 2006; 166:1822-1828.

19. Boult C, Green AF, Boult LB, Pacala JT, Snyder C, Leff B. Successful models of comprehensive care for older adults with chronic conditions: evidence for the Institute of Medicine's "Retooling for an aging America" report. J Am Geriatr Soc. 2009;57:2328-2337.

20. Meret-Hanke LA. Effects of the program of all-inclusive care for the elderly on hospital use. Gerontologist. 2011;51:774-785.

21. British Geriatrics Society. Fit for Frailty: Consensus Best Practice Guidance for the Care of Older People Living with Frailty in Community and Outpatient Settings. A Report by the British Geriatrics Society. London: British Geriatrics Society; 2014:1-26.

22. Jones DM, Song X, Rockwood K. Operationalizing a frailty index from a standardized comprehensive geriatric assessment. J Am Geriatr Soc. 2004;52:1929-1933.

23. Ritt M, Schwarz C, Kronawitter V, et al. Analysis of Rockwood Clinical Frailty Scale and Fried frailty phenotype as predictors of mortality and other clinical outcomes in older patients who were admitted to a geriatric ward. J Nutr Health Aging. 2015;19(10):1043-1048.
24. Rockwood K, Mitnitski A. Frailty defined by deficit accumulation and geriatric medicine defined by frailty. Clin Geriatr Med. 2011;27: 17-26.

25. Mahoney FI, Barthel DW. Functional evaluation: the Barthel Index. Md State Med J. 1965;14:61-65.

26. Salvi F, Morichi V, Grilli A, et al. Screening for frailty in elderly emergency department patients by using the Identification of Seniors At Risk (ISAR). J Nutr Health Aging. 2012;16(4):313-318.

27. Galea M, Woodward M. Mini-mental state examination (MMSE) Aust J Physiother. 2005;51(3):198.

28. Cullen B, O'Neill B, Evans JJ, Coen RF, Lawlor BA. A review of screening tests for cognitive impairment. J Neurol Neurosurg Psychiatry. 2007; 78(8):790-799.

29. Counsell SR, Callahan CM, Tu W, Stump TE, Arling GW. Cost analysis of the Geriatric Resources for Assessment and Care of Elders care management intervention. J Am Geriatr Soc. 2009;57(8):1420-1426.

30. Wong FK, Chow S, Chung L, et al. Can home visits help reduce hospital readmissions? Randomized controlled trial. J Adv Nurs. 2008;62(5): 585-595.

31. Cresswell JW. Research Design: Qualitative, Quantitative, and Mixed Methods Approaches. 4th ed. London: Sage publications; 2014.

32. Field A. Discovering Statistics Using IBM SPSS. 4th ed. London, UK: Sage Publications; 2013.

33. Stefanacci RG, Reich S, Casiano A. Application of PACE principles for population health management of frail older adults. Popul Health Manag. 2015;18(5):367-372.

34. Philp I, Mills KA, Thanvi B, Ghosh K, Long JF. Reducing hospital bed use by frail older people: results from a systematic review of the literature. Int J Integr Care. 2013;13(5) e048.

35. de Vries OJ, Peeters GM, Lips P, Deeg DJ. Does frailty predict increased risk of falls and fractures? A prospective population-based study. Osteoporos Int. 2013;24(9):2397-2403.

36. Kojima G. Frailty as a predictor of future falls among communitydwelling older people: a systematic review and meta-analysis. $J \mathrm{Am}$ Med Dir Assoc. 2015;16(12):1027-1033.
Clinical Interventions in Aging

\section{Publish your work in this journal}

Clinical Interventions in Aging is an international, peer-reviewed journal focusing on evidence-based reports on the value or lack thereof of treatments intended to prevent or delay the onset of maladaptive correlates of aging in human beings. This journal is indexed on PubMed Central, MedLine,

\section{Dovepress}

CAS, Scopus and the Elsevier Bibliographic databases. The manuscript management system is completely online and includes a very quick and fair peer-review system, which is all easy to use. Visit http://www.dovepress. com/testimonials.php to read real quotes from published authors. 